\title{
ESTUDIO DEL RIESGO FINANCIERO (5C) BAJO EL ENFOQUE DIFUSO
}

\author{
STUDY OF FINANCIAL RISK (5C) UNDER THE DIFFUSE APPROACH
}

Fecha de recepción: 2 de marzo de 2018. Fecha de aceptación: 12 de junio 2018.

Código JEL: E51, M21

Código DOI: $10.25097 /$ rep.n28.2018.04
Kléber A. Luna Altamirano

klunaa@ucacue.edu.ec

William H. Sarmiento Espinoza

wsarmiento@ucacue.edu.ec

Jaime Tinto Arandes

jtinto@ucacue.edu.ec

\section{Resumen}

Las empresas artesanales en la fabricación de muebles de madera de la ciudad de Cuenca-Ecuador, están sujetas al riesgo financiero empresarial dentro del sistema crediticio, el cual está sujeto a cumplir con las exigencias para la obtención de un crédito en una institución financiera. La metodología que se expone en el presente artículo, trata sobre utilización de las técnicas de las 5C (Carácter, Capital, Capacidad, Garantías Colaterales, Condiciones de la Economía) bajo el ámbito de la incertidumbre, con la aplicación de herramientas de avanzada de la lógica difusa como el expertizaje, cuyo objetivo es que estas empresas se sitúen en una adecuada categoría, y que la gerencia conozca si la institución financiera le puede conceder o no el respectivo crédito. El desarrollo de este estudio permite que toda organización tenga poder de decisión y previsión a la hora de acudir al financiamiento bancario.

Palabras clave: Riesgo financiero, técnicas de las $5 \mathrm{C}$, enfoque difuso.

\begin{abstract}
The artisanal companies of the city of Cuenca-Ecuador, it is the one related to the business financial risk within the credit system, which is subject to comply with the requirements for obtaining a credit in a financial institution. The methodology presented in this article deals with the use of $5 \mathrm{C}$ techniques (Character, Capital, Capacity, Collateral Guarantees, Conditions of the Economy) under the scope of uncertainty, with the application of advanced tools of the fuzzy logic as the expertization, whose objective is that the company is placed in an appropriate category, and that management knows whether the financial institution can grant or not the respective credit. The development of this study allows every organization to have decision and foresight power when it comes to bank financing.
\end{abstract}

Keywords: Financial risk, $5 \mathrm{C}$ techniques, diffuse approach. 


\section{Introducción}

Las pequeñas y medianas empresas, están sujetas a riesgos dentro de los mercado, donde las decisiones de obtener o no créditos comerciales, va a depender la actividad de la empresa. Este es el caso de las empresas artesanales fabricantes de muebles de madera de la ciudad de Cuenca-Ecuador, donde las finanzas dependen del otorgamiento o no de un crédito comercial que afecta la actividad y su desempeño. Es así, que la mayoría de empresarios desconoce los indicadores que la empresa debe alcanzar para ser evaluado por el ente crediticio y así obtener los requerimientos mínimos que exigen las entidades financieras en el otorgamiento de los créditos. En el caso de los propietarios de este tipo de empresas la solicitud de un crédito a la entidad bancaria, depende de la fragilidad con que dichas empresas se desarrollan a lo largo del año, enfrentándose a variables de alta volatilidad, que presentan altos niveles de desorden o entropía, que deberían ser controladas para obtener una respuesta acertada a la hora de solicitar financiamiento. La presente investigación se refiere a la aplicación de herramientas de avanzada de la lógica difusa, que permite atrapar los niveles de volatilidad de las variables por medio de rangos de valores que representen el recorrido de dichas variables a lo largo de un periodo en el tiempo y de esta forma estudiar la gestión del riesgo crediticio como elemento importante para los artesanos administradores en sus necesidades financieras.

En el desarrollo del estudio, se aplica la técnica de las 5C (Carácter, Capital, Capacidad, Garantías Colaterales, Condiciones de la Economía) bajo el ámbito de la incertidumbre, con el apoyo de las herramientas de avanzada como el expertizaje donde se propone un enfoque que recoge la variabilidad de cada una de dichas características en el tiempo, utilizando intervalos que permiten afirmar que la empresa se encuentra en una situación, entre una magnitud superior y una magnitud inferior, es decir, que la caracterización de las variables a estudiar están definidas dentro de un rango de acción, lo que llamaremos el intervalo de confianza, tal como lo presentan Kaufmann y Gil (1990), en su tratado sobre las matemáticas del azar y la incertidumbre. De esta forma se atrapa la incertidumbre de cada variable, entre rangos de acción para la toma de decisiones en la evaluación crediticia. Olarte (2006) asevera: "La incertidumbre es la inseguridad o duda que se tenga sobre el resultado de un acontecimiento futuro. A diferencia del riesgo, en la incertidumbre no se conoce la probabilidad de que ocurra el posible desenlace" (p.347).

Mediante un procedimiento iterativo, permite clasificar a cada empresa en una u otra categoría, cuyo objetivo es que la gerencia conozca si la institución financiera le puede conceder o no el respectivo crédito. El modelo presentado a los artesanos fabricantes de muebles de madera, constituye un apoyo para realizar un análisis financiero interno dentro de la empresa antes de acudir a la entidad bancaria a solicitar un crédito, con el propósito de que la solicitud sea aceptada en las mejores condiciones. El resultado es el análisis de las cinco variables mediante intervalos de confianza, que permita atrapar la información de los expertos analistas bancarios y administradores de las empresas, por medio de bandas que representan el umbral de posibilidad de pasar a un nivel superior del nivel de aceptación de un crédito. En el caso de llegar la empresa artesanal a un nivel bajo, deberá entrar en un plan de mejora, en cuanto a la característica que este fallando, con el propósito de lograr un nivel óptimo aceptable. Con ello los artesanos fabricantes de este tipo de producto, tendrán poder de decisión y previsión a la hora de acudir al financiamiento bancario, pre evaluándose para conocer en qué medida y condición podrá obtener el crédito.

\section{Estado del arte}

El riesgo de no obtener créditos financieros está presente en la mayor parte de las empresas. 
No escapan de ello las empresas artesanales fabricantes de muebles de madera de la ciudad de Cuenca-Ecuador, por no cubrir las exigencias crediticias por parte de las entidades financieras para el otorgamiento de un crédito comercial. En lo referente al riesgo, Olarte (2006) sostiene: "Es el grado de variabilidad o contingencia del retorno de una inversión. En términos generales se puede esperar que, a mayor riesgo, mayor rentabilidad de la inversión" (p.348). De Lara (2005) sostiene:

La medición efectiva y cuantitativa del riesgo se asocia con la probabilidad de una pérdida en el futuro. Los seres humanos deben conocer y responder de manera intuitiva o cuantitativa a las probabilidades que confrontan en cada decisión. La esencia de la administración de riesgos consiste en medir esas probabilidades en contextos de incertidumbre. (p.13)

Herrera y Terán (2008) afirman: "El riesgo financiero es la posibilidad de que se produzca un hecho generador de pérdidas que afecten el valor económico de las instituciones. Dicho de otra manera, es la probabilidad de sufrir una pérdida de valor económico" (p.142). Para tener una idea más concreta se dice que el riesgo financiero es el resultado del dinero prestado a una persona natural o empresa, lo cual conlleva a un conflicto de que el deudor no cumpla con los pagos de la deuda adquirida. Arias, Rave y Castaño (2006) aseveran:

El riesgo financiero se define como "la incertidumbre asociada con el valor y/o retorno de una posición financiera". La incertidumbre no es más que una situación general de desconocimiento del futuro, mientras que el riesgo, es la probabilidad de que ocurra un evento desfavorable. EI riesgo está ligado a la incertidumbre sobre eventos futuros, lo que hace que resulte imposible eliminarlo por completo, por ello se deben elegir las mejores estrategias para tratar de controlarlo. (p.275)

Todo riesgo genera incertidumbre, por ello es necesario estudiar la gestión de riesgos. Herrera y Terán (2008) sostienen: "La gestión de riesgos es la denominación que se utiliza para el conjunto de técnicas y herramientas que pretenden maximizar el valor económico de la institución financiera, en un contexto de incertidumbre" (p.143). La gestión de riesgos es el resultado de la evaluación de riesgos por la aplicación de procesos reglamentarios y normativos. Otros autores han publicado sus estudios sobre riesgos financieros como: Sarmiento y Vélez (2007). Teoría del riesgo en mercados financieros: una visión teórica. Cairó y Gómez (2015). El enfoque financiero vs. El enfoque social del microcrédito. Un análisis comparativo mundial. Torres y Olarte ((2009). Valor en riesgo desde un enfoque de cópulas. Hammi (2014). Control financiero interno bajo incertidumbre: Control de la gestión de la liquidez.

Lotfi Zadeh profesor de la Universidad de Berkeley, en 1975 publicó su trabajo "Fuzzy Sets", en el cual se describen los fundamentos matemáticos agregados a la teoría de conjuntos difusos, dando inicio a la lógica difusa. Algunos autores han afirmado los conceptos teóricos relacionado a la incertidumbre, a través de la lógica borrosa también llamada lógica difusa. Reig y González (2002) indican: "la lógica borrosa se revela como un instrumento muy potente (...) al permitir, por un lado, recoger la incertidumbre generada por el entorno de la empresa, y por otro tratar la subjetividad que implica toda opinión de expertos" (p.436). Domínguez, Ruiz y Sánchez (1992) definen: "Un número borroso es la asociación de dos conceptos, el de intervalos de confianza ligado, a la incertidumbre y el de nivel de presunción, ligado a la subjetividad" (p.49). A la incertidumbre se le califica como un vacío dentro de la seguridad, es decir el desconocimiento sobre un tema. Ramírez (1998), (citado por Mongrut y Ramírez, 2004) manifiestan que la incertidumbre es un estado de duda frente a una determinada situación cuyos resultados, causas o características no se conocen en el momento presente o frente a la cual no se sabe cómo actuar; por ello (Aguiar, 2004) señala que las situaciones de incertidumbre se caracterizarían por el hecho de que no sólo se desconoce el resultado final, sino que, no se puede predecirlo tampoco en términos de probabilidades objetivas. La lógica difusa, permite utilizar conceptos relativos de la realidad, definiendo grados variables 
de pertenencia y siguiendo patrones de razonamiento similares a los del pensamiento humano (Kosko, 1995). Kaufmann y Gil (1987) en su obra "Técnicas operativas de gestión para el tratamiento de la incertidumbre", conceptúan a un número borroso como una secuencia finita o infinita de intervalos de confianza, estudio que ha brindado un significativo aporte al conocimiento científico.

Casanovas y Fernández (2003) en su obra la gestión de la tesorería en la incertidumbre, proponen una técnica de avanzada relacionada a la aplicación de las 5C (carácter, capital, capacidad, garantías colaterales y condiciones), la cual consiste en evaluar estas condiciones a las distintas empresas o clientes, pudiendo llegar a tomar una decisión de concesión o rechazo de un crédito bancario, por parte de una institución financiera. Los mencionados autores entregan un gran aporte científico mediante la gestión de tesorería moderna, plantean herramientas de vanguardia, que son valoradas por quienes deseen generar una eficiente dirección empresarial.

\section{Metodología}

Para determinar la calidad crediticia de las empresas artesanales fabricantes de muebles de madera de la ciudad de Cuenca-Ecuador, se procede a analizar las cinco variables importantes al momento de solicitar un crédito bancario a una entidad financiera, para ello se tiene que definir cada uno de estos factores. Casanovas y Fernández (2003) afirman:

Carácter: se refiere a la filosofía y ética del cliente como pagador (...). Capital: se refiere a la solvencia del cliente, concretamente a los fondos propios de los que dispone para hacer frente a los problemas financieros que puedan surgir. Capacidad: (...) capacidad que tiene la empresa para generar liquidez suficiente y así hacer frente a los pagos (...). Garantías colaterales: se refiere a todos aquellos activos de los que dispone el cliente como garantía del crédito obtenido (...). Condiciones: se trata de evaluar la situación de la economía general del momento, así como la sensibilidad del flujo de tesorería y de los ingresos de explotación ante variaciones de esta (...). (p.299)

Este método, sugiere trabajar con la aplicación de las $5 \mathrm{C}$ bajo un entorno incierto, donde cada variable será evaluada por un conjunto de expertos que permiten atrapar mediante intervalos, las tendencias de cada variable, durante un periodo de tiempo estudiado.

El modelo está basado en la construcción de un grafo, como se muestra en la figura 1. Para el desarrollo y aplicación de las $5 \mathrm{C}$ se recurre en primer lugar a un grupo de expertos, quienes evaluaran la variable "carácter" del cliente, por medio de la escala semántica endecadaría, presentada en la tabla 1. Una vez obtenida la valoración correspondiente se clasifica al cliente, como fuerte $(F)$ o débil $(D)$, según la evaluación supere o no, el umbral prefijado, por los expertos crediticios de las entidades. Se repite este proceso continuamente bajo el camino estructurado por el grafo para el resto de variables. Hasta calificar al cliente en ocho grupos, donde el grupo 1 representa el mejor posesionado y el grupo 8 el peor respecto a la calidad crediticia.

Luego, definimos una política de crédito para cada uno de los 8 grupos, por ejemplo, si la puntación es 1 (grupo 1) se decidirá conceder el crédito al cliente en la cuantía máxima posible, mientras que si es 8 (grupo 8) se le denegara dicho crédito exigiendo condiciones muy estrictas en caso de concedérselo en lo referente a los pagos.

Entre los extremos de los grupos se definirá toda una gama de políticas de crédito atendiendo al riesgo, del cliente a evaluar (empresa artesanal del mueble).

Con la aplicación de este instrumento se podrá ver la calidad en la orientación crediticia de 
la empresa a lo largo del tiempo y repitiendo este procedimiento con cierta periodicidad, permitirá al empresario saber si esta en condiciones de solicitar incrementos crediticios o nuevos créditos.

En este estudio se cuenta con el aporte de la técnica de avanzada del expertizaje, la cual ayudará a reducir la incertidumbre en la determinación de los umbrales de cada una de las variables, para la clasificación a cada empresa artesanal en una u otra categoría, esto permitirá que el artesano propietario conozca si la entidad financiera le puede conceder o no el crédito solicitado.

\subsection{Teoría del expertizaje}

Levantar información a un grupo de expertos, se conoce como expertizaje, relacionado a un tema específico, con la finalidad de disminuir la incertidumbre. Experto es toda persona con habilidades y destrezas y apropiadamente capacitado en un tema objeto de consulta gracias a la experiencia empírica, profesional o académica (Medina, 2006). Se obtiene información a los agentes de crédito de las diferentes entidades financieras de la ciudad de Cuenca, con la finalidad de determinar los umbrales para cada una de las variables, además se cuenta con la consulta a un grupo de expertos de las diferentes empresas artesanales, quienes calificarán por medio de la escala endecadaria ( tabla 1), asignando valores a cada una de las variables concernientes a las $5 \mathrm{C}$, utilizando intervalos de confianza (bandas), para determinar el mejor y peor de los escenarios que se le pudieran presentar al empresario.

La herramienta del expertizaje es una de las más utilizadas dentro de la lógica difusa, la escala endecadaria reduce la entropía y ajusta los valores examinados. Kaufmann y Gil-Aluja (1989) expresan: "La introducción de una valuación matizada entre 0 y 1 permite hacer intervenir niveles de verdad en la noción de incidencia. (...) Valores de 0 a 1 (la llamada valuación endecadaria)" (p.26). La escala endecadaria o semántica se presenta en tabla 1.

Tabla 1. Escala endecadaria

\begin{tabular}{|l|l|}
\hline GRADO DE PRESUNCIÓN $\alpha$ & INCIDENCIA \\
\hline 0 & No tiene influencia. \\
\hline 0,1 & Tiene mínima influencia. \\
\hline 0,2 & Tiene poca influencia. \\
\hline 0,3 & Tiene algo de influencia. \\
\hline 0,4 & Tiene una influencia regular \\
\hline 0,5 & $\begin{array}{l}\text { Puede influir como puede no } \\
\text { influir }\end{array}$ \\
\hline 0,6 & Tiene bastante influencia. \\
\hline 0,7 & Tiene una importante influencia. \\
\hline 0,8 & Tiene mucha influencia. \\
\hline 0,9 & Tiene muchísima influencia. \\
\hline 1 & Máxima influencia. \\
\hline
\end{tabular}

Elaboración y fuente: los autores 
Basándose en la escala endecadaria enunciada, se solicita a los profesionales del departamento de crédito de las diferentes instituciones financieras de la ciudad de Cuenca-Ecuador, determinen la importancia que tiene cada variable (componentes $5 \mathrm{C}$ ), como calificación para otorgar el crédito a las empresas artesanales solicitantes. Los resultados obtenidos en lo que respecta a la variable Carácter, de cinco expertos en el área de crédito son los siguientes:

Experto no.1: 0,9

Experto no.2: 0,7

Experto no. 3: 0,8

Experto no. 4: 0,8

Experto no. 5: 0,9

Como se puede observar, 0,7 se repite una vez, 0,8 y 0,9 se repiten dos veces. Se anota el total de veces que se reitera en la escala endecadaria las opiniones de los cinco expertos, relacionado únicamente con la primera variable. El siguiente paso es normalizar la serie de esta herramienta, el cual consiste en dividir el número de veces que se repite correspondiente a la calificación con relación a la escala endecadaria entre el número de expertos (5), de la siguiente manera: $1 \div 5=0,2 ; 2 \div 5=0,4 ; y, 2 \div 5=0,4$, así sucesivamente. Luego se procede a acumular desde el final de la serie hasta que se obtiene el valor de la unidad, de ahí en adelante todos los valores serán uno. Para finalizar con la aplicación de esta herramienta se procede a realizar la sumatoria de la acumulación de frecuencias, sin tomar en consideración el grado de presunción a igual a cero, el resultado se indica en la tabla 2.

Tabla 2. Serie normalizada y acumulación de frecuencias

\begin{tabular}{|l|l|l|l|}
\hline $\begin{array}{l}\text { GRADO DE PRE- } \\
\text { SUNCIÓN } \alpha\end{array}$ & FRECUENCIA & $\begin{array}{l}\text { NORMALIZACIÓN } \\
\text { DE LA FRECUENCIA }\end{array}$ & $\begin{array}{l}\text { ACUMULACIÓN DE } \\
\text { FRECUENCIAS }\end{array}$ \\
\hline 0 & 0 & 0 & 1 \\
\hline 0,1 & 0 & 0 & 1 \\
\hline 0,2 & 0 & 0 & 1 \\
\hline 0,3 & 0 & 0 & 1 \\
\hline 0,4 & 0 & 0 & 1 \\
\hline 0,5 & 0 & 0 & 1 \\
\hline 0,6 & 0 & 0 & 1 \\
\hline 0,7 & $1-$ May & 0,2 & 1 \\
\hline 0,8 & $2-$ May & 0,4 & 0,8 \\
\hline 0,9 & $2-$ May & 0,4 & 0,4 \\
\hline 1 & 0 & 0 & 0 \\
\hline TOTAL & 5 & 1 & 8,2 \\
\hline & & & \\
\hline
\end{tabular}

Elaboración y fuente: los autores

El total de la acumulación de frecuencias debe dividirse entre 10 que corresponde al número de factores que forman el grado de presunción, tomando en consideración desde 0,1 hasta 1, cuyo resultado es igual a $8,2 \div 10=0,8$. Análogamente se aplica la misma técnica para el resto de variables que conforman las $5 \mathrm{C}$, dando el siguiente resultado. 
Tabla 3. Determinación de los Umbrales

\begin{tabular}{|l|l|}
\hline VARIABLES & UMBRALES \\
\hline Carácter & 0,8 \\
\hline Capital & 0,7 \\
\hline Capacidad & 0,8 \\
\hline Garantías Colaterales & 0,9 \\
\hline Condiciones de la Economía & 0,7 \\
\hline
\end{tabular}

Elaboración y fuente: los autores

Posterior a ello, se levanta información a las personas expertas en la parte financiera de una empresa artesanal fabricantes de muebles de madera de la ciudad de Cuenca, quienes califican asignando un valor cuantitativo a cada uno de las variables, considerando una opinión pesimista y otra optimista, en intervalos de confianza (bandas), concernientes a las 5C.

Se recepta la información a cuatro expertos con respecto a la variable "Carácter", concerniente a la percepción de ellos con relación a la importancia de esta variable, para la obtención de un crédito bancario. Los resultados son los siguientes:

Experto no. 1: $[0,8,0,8]$

Experto no. 2: $[0,8,1,0]$

Experto no. 3: $[0,9,0,9]$

Experto no. 4: $[0,7,0,9]$

El procedimiento para la aplicación del expertizaje con intervalos de confianza, es el mismo que el caso anterior, la aplicación de esta herramienta de avanzada se presenta a continuación:

Tabla 4. Serie normalizada y acumulación de frecuencias (Bandas)

\begin{tabular}{|l|l|l|l|l|l|l|}
\hline $\begin{array}{l}\text { GRADO DE PRE- } \\
\text { SUNCIÓN } \alpha\end{array}$ & \multicolumn{2}{l|l}{ FRECUENCIA } & \multicolumn{2}{l|}{$\begin{array}{l}\text { FRECUENCIAS NOR- } \\
\text { MALIZADAS }\end{array}$} & \multicolumn{2}{l|}{$\begin{array}{l}\text { EXPERTON CAPACI- } \\
\text { DAD }\end{array}$} \\
\hline 0 & 0 & 0 & 0 & 0 & 1 & 1 \\
\hline 0,1 & 0 & 0 & 0 & 0 & 1 & 1 \\
\hline 0,2 & 0 & 0 & 0 & 0 & 1 & 1 \\
\hline 0,3 & 0 & 0 & 0 & 0 & 1 & 1 \\
\hline 0,4 & 0 & 0 & 0 & 0 & 1 & 1 \\
\hline 0,5 & 0 & 0 & 0 & 0 & 1 & 1 \\
\hline 0,6 & 0 & 0 & 0 & 0 & 1 & 1 \\
\hline 0,7 & 1 & 0 & 0,25 & 0 & 1 & 1 \\
\hline 0,8 & 2 & 1 & 0,5 & 0,25 & 0,75 & 1 \\
\hline 0,9 & 1 & 2 & 0,25 & 0,5 & 0,25 & 0,75 \\
\hline 1 & 0 & 1 & 0 & 0,25 & 0 & 0,25 \\
\hline TOTAL & 4 & 4 & & & 8 & 9 \\
\hline
\end{tabular}

Elaboración y fuente: los autores 
El expertón encontrado se debe dividirse entre 10, como ya se explicó anteriormente, los resultados en bandas son $[0,8,0,9]$. Análogamente se aplica la misma técnica para el resto de variables, posterior a ello, se analiza los resultados de cada una de las variables, con relación a los umbrales obtenidos en la tabla 3 , determinando si superan o no estos umbrales, luego se clasifican a cada variable en base a dos indicadores cuyos nombres se denominarán como fuerte o débil, lo explicado se detalla en la siguiente tabla.

Tabla 5. Determinación de los expertones e indicadores

\begin{tabular}{|l|l|l|l|}
\hline \multirow{2}{*}{ VARIABLES } & \multirow{2}{*}{ UMBRALES } & \multicolumn{1}{|c|}{ INTERVALOS DE CONFIANZA } & \\
\cline { 3 - 4 } & & \multicolumn{1}{|c|}{ (Expertón) } & \multicolumn{1}{c|}{ INDICADORES } \\
\hline Carácter & 0,8 & {$[0,8,0,9]$} & Fuerte \\
\hline Capital & 0,7 & {$[0,8,0,9]$} & Débil \\
\hline Capacidad & 0,8 & {$[0,8,0,9]$} & Fuerte \\
\hline $\begin{array}{l}\text { Garantías Cola- } \\
\text { terales }\end{array}$ & 0,9 & {$[0,8,0,9]$} & Fuerte \\
\hline $\begin{array}{l}\text { Condiciones de } \\
\text { la Economía }\end{array}$ & 0,7 & {$[0,8,0,9]$} & Débil \\
\hline
\end{tabular}

Elaboración y fuente: los autores

A continuación, se procede a construir un grafico en el cual se seguirá la ruta trazada en base a los indicadores encontrados para cada una de las variables, correspondiente a la técnica de las $5 \mathrm{C}$, de esta manera se determinará la calidad crediticia de la empresa artesanal, a continuación, se presenta la estructura de esta malla, siguiendo el camino marcado en relación a lo indicado.

Gráfico 1. Grafo ruta de aplicación de la técnica de las $5 \mathrm{C}$

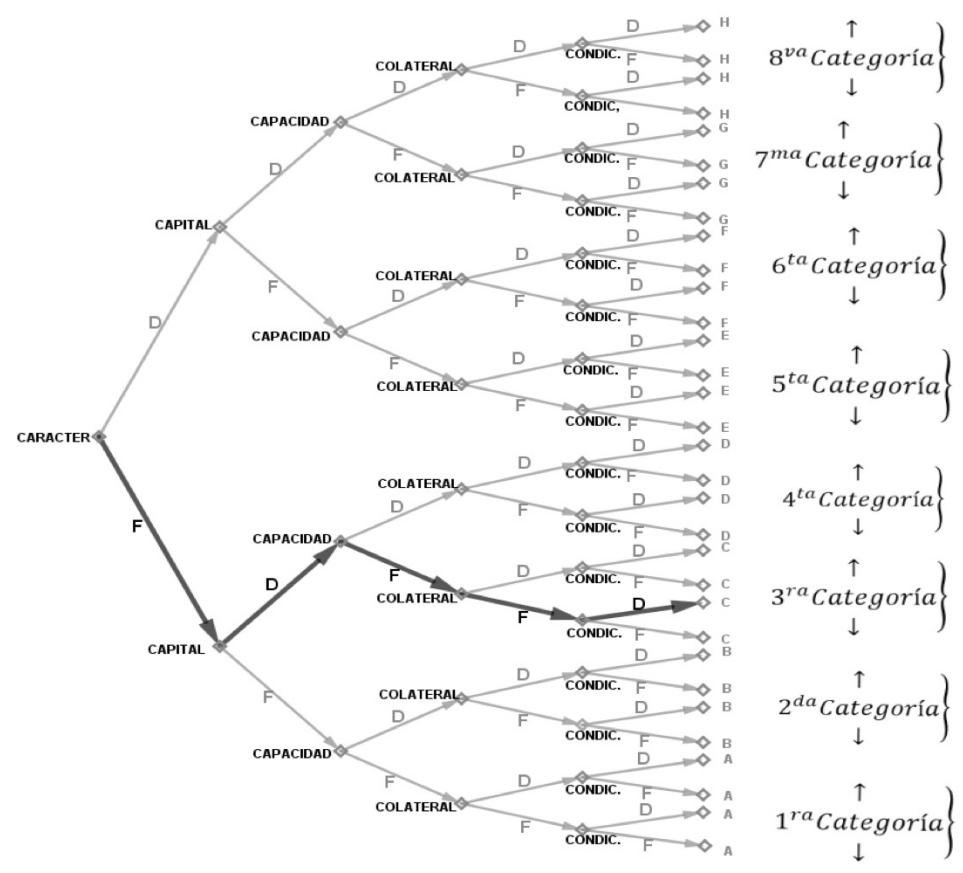

Elaboración y fuente: los autores 


\section{Resultados y Discusión}

Siguiendo la ruta del grafo con relación a las cinco variables, se obtiene la valoración correspondiente, clasificando al cliente como fuerte o débil, cabe explicar que dentro de esta clasificación se determinan ocho categorías, siendo la categoría 1 y la categoría 8 la aceptable y la denegada respectivamente, en lo que se refiere a la calidad crediticia. El resultado obtenido de la investigación, sitúa en la categoría 3 a la empresa artesanal fabricante de muebles de madera de acuerdo a una gama de políticas de crédito por parte de la institución financiera. En el presente estudio la empresa estaría dentro del rango de aceptable para la aprobación de la solicitud de crédito. Si se llegaría a obtener una categorización más baja y no admisible, la empresa deberá entrar en un plan de mejora, entendiéndose que la mejora consiste en corregir las variables deficientes, convirtiéndolas en fortalezas con el propósito de llegar a alcanzar tal objetivo en una fecha inmediata.

\section{Conclusiones}

La aplicación de esta herramienta de avanzada, consiste en evaluar a cada una de las cinco variables (Carácter, Capital, Capacidad, Garantías Colaterales, Condiciones de la Economía), con ello se permite llegar a una deducción sobre el perfil de la empresa artesanal fabricante de muebles de madera de la ciudad de Cuenca-Ecuador, con el propósito de determinar si cumple o no con los requerimientos de la institución financiera, para la concesión o rechazo del crédito comercial. Esta técnica de las $5 \mathrm{C}$, propone un nuevo enfoque bajo incertidumbre, el cual analiza la calidad crediticia de cualquier cliente o empresa a partir del análisis de estas cinco variables, valorando las mismas, se llega a obtener el riesgo crediticio antes de proceder a solicitar un crédito bancario, con ello se pretende entregar a este tipo de empresa artesanal, esta herramienta de vanguardia que ofrece la lógica difusa, para llegar a tomar la mejor decisión antes de solicitar un crédito en cualquier institución financiera. 


\section{Referencias bibliográficas}

Aguiar, F. (2004). Teoría de la decisión e incertidumbre: modelos normativos y descriptivos. Empiria. Revista De Metodología De Ciencias Sociales, 8, 139-160. Recuperado de http://dx.doi.org/10.5944/ empiria.8.2004.982

Arias, L., Rave, S., y Castaño, J. (2006). Metodologías para la medición del riesgo financiero en inversiones. Scientia Et Technica, XII (32), 275-278. Recuperado de http://www.redalyc.org/articulo.oa?id=84911652048

Cairó, G., y Gómez, L. (2015). El enfoque financiero vs. El enfoque social del microcrédito. Un análisis comparativo mundial. REVESCO. Revista De Estudios Cooperativos, 118, 31-59. Recuperado de https:// revistas.ucm.es/index.php/REVE/article/view/49062

Casanovas, M., y Fernández, A. (2003). La gestión de la tesorería en la incertidumbre. Madrid, España: Ediciones Pirámide.

De Lara, A. (2005). Medición y control de riesgos financieros. Recuperado de https://books.google.com. ec/books/about/Medici\%C3\%B3n_y_control_de_riesgos_financier.html?id=PrQ-vTEWLqoC\&redir_esc=y

Domínguez, M., Ruiz, M., y Sánchez, J. (1992). Valoración de rentas de capital con tipos de interés borroso. Cuadernos de Estudios empresariales, 2, 47-55. Recuperado de https://dialnet.unirioja.es/servlet/ articulo?codigo $=164199$

Hammi, A. (2014). Control financiero interno bajo incertidumbre: Control de la gestión de la liquidez (tesis doctoral). Universidad de Barcelona, España.

Herrera, M., y Terán, J. (2008). Conceptualización del riesgo en los mercados financieros. Revista de Derecho, 10, 141-155. Recuperado de http://revistas.uasb.edu.ec/index.php/foro/article/view/359/356

Kaufmann, A., y Gil-Aluja, J. (1989). Modelos para la investigación de efectos olvidados. Barcelona, España: Milladoiro.

Kaufmann, A., y Gil-Aluja, J. (1987). Técnicas operativas de gestión para el tratamiento de la incertidumbre. Barcelona, España: Hispano Europea

Kaufmann A., y Gil Aluja , J.(1990). Las matemáticas del azar y de la incertidumbre. Elementos básicos para su aplicación en economía. Madrid, España: Centro de estudios Ramón Areces.

Kosko, B. (1994). Fuzzy systems as universal approximators. IEEE Transactions on Computers, 43(11), 1329-1333. Recuperado de http://sipi.usc.edu/ kosko/FuzzyUniversalApprox.pdf

Medina, S. (2006). Estado de la cuestión acerca del uso de la lógica difusa en problemas financieros. 
Cuadernos de Administración, 32(19), 195-223. Recuperado de http://www.scielo.org.co/pdf/cadm/v19n32/ v19n32a09.pdf

Mongrut, S., y Ramírez, D. (2014). Análisis financiero en incertidumbre: una propuesta metodológica. Apuntes: Revista De Ciencias Sociales, 0(54), 5-25. Recuperado de http://revistas.up.edu.pe/index.php/ apuntes/article/view/212

Olarte, J. (2006). Incertidumbre y evaluación de riesgos financieros. Scientia Et Technica, XII (32), 347350. Recuperado de http://www.redalyc.org/articulo.oa?id=84911652061

Ramírez, D. (1988). Fundamentos metodológicos para el análisis económico en contexto de incertidumbre. Universidad de Barcelona, España.

Reig, J., y González, J. (2002). Modelo borroso de control de gestión de materiales. Revista Española de Financiación y Contabilidad, 31(112), 431-459. Recuperado de https://www.jstor.org/ stable/42781484?seq=1\#page_scan_tab_contents

Sarmiento, R., y Vélez, R. (2007). Teoría del riesgo en mercados financieros: una visión teórica. Cuadernos Latinoamericanos de Administración, III (4), 25-49. Recuperado de http://www.redalyc.org/articulo. oa? id=409634347003

Torres, G., y Olarte, A. (2009). Valor en riesgo desde un enfoque de cópulas. AD-minister, (15), 113-136. Recuperado de http://www.redalyc.org/articulo.oa?id=322327246006 